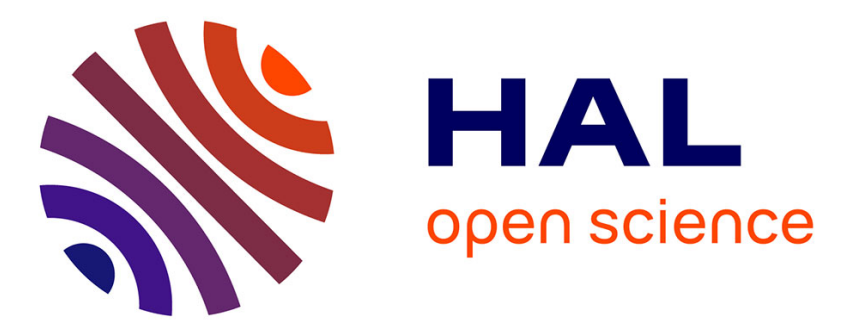

\title{
Terminologie de la qualité pour la modélisation numérique en géotechnique: discussion
}

Véronique Merrien-Soukatchoff, Yvon Riou

\section{To cite this version:}

Véronique Merrien-Soukatchoff, Yvon Riou. Terminologie de la qualité pour la modélisation numérique en géotechnique: discussion. Revue Française de Génie Civil , 1999, 3 (7-8), pp.481-494. 10.1080/12795119.1999.9692657 . hal-01448196

\section{HAL Id: hal-01448196 \\ https://hal.science/hal-01448196}

Submitted on 27 Jan 2017

HAL is a multi-disciplinary open access archive for the deposit and dissemination of scientific research documents, whether they are published or not. The documents may come from teaching and research institutions in France or abroad, or from public or private research centers.
L'archive ouverte pluridisciplinaire HAL, est destinée au dépôt et à la diffusion de documents scientifiques de niveau recherche, publiés ou non, émanant des établissements d'enseignement et de recherche français ou étrangers, des laboratoires publics ou privés. 


\title{
Terminologie de la qualité pour la modélisation numérique en géotechnique : discussion
}

\section{Véronique Merrien-Soukatchoff* — Yvon Riou**}

\author{
* LAEGO-Ecole des Mines de Nancy, Parc de Saurupt, F-54042 Nancy cedex \\ ** LGCNSN - Ecole Centrale de Nantes, BP 92101, F-44321 Nantes cedex 03
}

\begin{abstract}
RÉSUMÉ. Depuis que les méthodes numériques existent, des progrès significatifs ont permis de s'affranchir d'un certain nombre d'hypothèses simplificatrices dans le calcul des ouvrages de géotechnique. Les modélisations étant de plus en plus sophistiquées et en l'absence d'un cadre réglementaire, il est apparu nécessaire de s'intéresser à leur qualité. Mais avant de s'engager dans la qualité et l'assurance qualité, une synthèse des pratiques actuelles en matière de terminologie nous a paru utile. Le présent document liste des termes relevant de la qualité en précisant leur interprétation dans le domaine de la modélisation numérique en géotechnique, puis des termes, employés dans le domaine de la modélisation en géotechnique, que l'on peut rattacher au concept de qualité. Pour ces derniers nous avons recensé la définition proposée par les normes relatives à la qualité ou par des dictionnaires, et l'utilisation qui en est faite par différents auteurs. Sur la base de ces informations, nous avons soumis des propositions ou apporté des éléments de réflexion quant à l'utilisation de ces termes. Cette synthèse devrait contribuer à pratiquer un langage commun dans le domaine de la modélisation numérique du moins en géotechnique, et à distinguer les différentes notions relevant de la qualité.
\end{abstract}

ABSTRACT. From the beginning of numerical methods, because of several significant improvements, geotechnical engineers no longer need the use of simplifying hypotheses. If theses new techniques for modelling soil structures are attractive, one can fear, because of sophistication, that engineers do not fully understand the code. Then, it seems necessary to establish a system for quality of various basic steps of modelling, and for the coherence of sequence of steps. But, before getting involved in quality system, we consider that a synthesis of actual terminological practice would be useful. We propose, herein, various quality notions. We hope this document will emerge on common word-list in civil engineering modelling.

MoTS-CLÉS : méthodes numériques, géotechnique, qualité, assurance qualité, lexique.

KEYWORDS: numerical methods, geotechnics, quality, quality assurance, lexico. 


\section{Introduction}

Tous les intervenants dans l'étude d'un ouvrage de géotechnique ont le souci du « travail bien fait» : du concepteur de code au concepteur de modèle, de l'ingénieur informaticien à l'ingénieur bureau d'étude. Chacun dans son domaine cherche en permanence à parfaire sa connaissance de modélisateur, ses outils de dimensionnement, son expérience de terrain... Dans le domaine de la géotechnique, cela comprend la connaissance du site, la connaissance des matériaux, la connaissance du mode de fonctionnement de l'ouvrage, la conception du' modèle, sa mise en cuvre dans un code de calcul, le contrôle de ce code, l'ajustement des paramètres, le contrôle du modèle, l'interprétation des résultats...

Depuis que les méthodes numériques existent, des progrès significatifs ont permis de s'affranchir d'un certain nombre d'hypothèses simplificatrices. Par exemple, la géométrie de l'ouvrage n'est plus nécessairement plane, le matériau n'est plus simplement élastique linéaire, les équations d'équilibre et de diffusion ne sont plus traitées séparément quand les phénomènes mécaniques et hydrauliques sont en interaction. Si nous pouvons nous rejouir des nouveaux moyens à notre disposition pour traiter les diverses étapes de simulation du comportement d'un ouvrage dans son environnement, on peut craindre, en raison de la complexité qui en résulte, que l'utilisateur ne maîtrise pas parfaitement l'exploitation du code de calcul.

Nous pouvons, par conséquent, légitimement nous interroger sur la qualité des modélisations (Peut-on la contrôler, l'évaluer ? Est-il nécessaire (et possible) de définir des règles communes pour le calcul de tous les ouvrages ?). Le souci de qualité n'est pas nouveau dans le domaine du Génie Civil puisque des démarches ont été initiées pour des types particuliers d'ouvrages, notamment les barrages [CIG 94]. Il semble cependant que la qualité de la modélisation numérique en Génie Civil n'ait pas été abordée de manière à la fois globale et spécifique à ce thème. Il paraît donc utile de proposer un système qualité spécifique, généralisant les principes formulés pour certains types d'ouvrage et déclinant les principes généraux issus du management de la qualité.

Le manque d'approche globale mentionné plus haut conduit à un manque de consensus sur les termes définissant la qualité et ce manque de consensus sur la terminologie est préjudiciable à la qualité elle-même. Nous proposons donc dans ce qui suit un inventaire de termes relatifs à la qualité dans une modélisation numérique en géotechnique.

Deux types de termes sont associés à cette modélisation en géotechnique :

- ceux relevant de la qualité, que nous traiterons en partie dans ce qui suit, en précisant la manière dont ils peuvent être interprétés pour notre problématique ; 
- ceux plus spécifiques à la modélisation numérique en géotechnique, que l'on peut rattacher au concept de qualité.

Avant tout, nous précisons que nous emploierons, conformément à la norme, le terme code de calcul pour désigner les logiciels, composés de programmes, avec lesquels la modélisation est effectuée.

\section{Termes courants de la qualité}

Il serait bien trop ambitieux de traiter tous les aspects de la qualité dans ce paragraphe. Aussi, nous restreindrons notre propos à son contrôle, celui qui permet d'assurer la conformité du " produit " Nous n'aborderons pas ici les notions telles que la qualité totale, la politique qualité, les actions préventives, l'auto-évaluation, l'anticipation de la qualité, l'élaboration de la documentation spécifique à la qualité, la certification... actions qui se situent soit en amont de notre préoccupation actuelle (engagement de la qualité), soit en aval (amélioration de la qualité, certification).

\subsection{Qualité}

D'après la norme ISO 8402-2.1 [AFN 96], c'est « l'ensemble des caractéristiques d'une entité qui lui confêre l'aptitude à satisfaire des besoins exprimés et implicites ». Les entités concernées, dans notre cas, peuvent être le code de calcul ou la note de calcul selon le client envisagé (utilisateur du code ou destinataire de la note de calcul), ou plus généralement l'étude qui inclut un calcul numérique. La qualité peut comprendre : les fonctionnalités, la performance, la facilité d'emploi, la sûreté de fonctionnement, la sécurité, la fiabilité, le coût, les délais, les services liés aux produits...

La qualité se décompose classiquement en qualité externe qui vise le produit en soi (code, note de calcul) et qualité interne qui s'intéresse aux processus. Dans le cas présent, la qualité interne concerne :

- les processus intervenant dans les étapes d'élaboration du code (analyse des besoins, définition du projet, organisation, programmation, contrôle du code, documentation) ;

- les processus de qualification des utilisateurs du code, du moins en partie ;

- les processus d'exploitation du code qui incluent les différentes phases de modélisation (choix des hypothèses, de la méthode de résolution, du code, de la géométrie, des modèles de comportement, du chargement, de la discrétisation... vérification des données et des résultats). 


\subsection{Système qualité}

C'est, d'une manière générale, l'ensemble des ressources qui participent aux objectifs qualité de l'entreprise (structure organisationnelle, responsabilités, procédures, procédés). D'une manière plus restrictive, le système qualité peut désigner la structure et les procédures mises en place pour mettre en cuvre le management de la qualité.

\subsection{Maîtrise de la qualité}

Ce sont les techniques et activités à caractère opérationnel qui ont pour but de piloter les processus et d'éliminer les dysfonctionnements à toutes les étapes de la "fabrication " (mise en cuvre du code ou de la modélisation). En ce qui concerne le code de calcul, ces techniques sont largement commentées dans les documents de génie logiciel et d'analyse numérique.

\subsection{Contrôle de la qualité}

D'après la norme ISO 8402-2.1 [AFN 96], le contrôle de la qualité consiste à " mesurer, examiner une ou plusieurs caractéristiques d'une entité et comparer les résultats aux exigences spécifiées en vue de déterminer si la conformité est obtenue pour chacune des caractéristiques ».

Il s'agit, notamment pour le code, du contrôle opérationnel (le code est conforme au cahier des charges), et du contrôle fonctionnel (les opérations prévues sont effectuées avec succès par le code). Concernant la note de calcul, le contrôle qualité n'est pas réellement formalisé, sauf pour certains ouvrages sensibles (nucléaire...). Cette opération doit être effectuée en interne par le concepteur et l'utilisateur du code, mais également en externe. Par ailleurs, cette opération est délicate, car les exigences en matière de calcul numérique ne sont pas toujours bien formulées par les « clients » (utilisateurs du code ou destinataires de la note de calcul).

\subsection{Assurance qualité}

L'assurance qualité vise à donner confiance au «client». Elle passe par des dispositifs pris par le fournisseur pour prouver qu'il satisfait les exigences pour la qualité, mais sans signifier que les produits sont nécessairement bons. Dans le domaine de la modélisation numérique, elle s'applique au code, à la méthode de modélisation elle-même et à l'application particulière. Les dispositifs attestant d'une certaine confiance, peuvent être des documents (manuel d'assurance qualité, manuel d'exemples avec cas tests, manuel informatique pour le concepteur de code), des 
actions de veille technologique et de formation, la participation à des benchmarks. Il est bien clair, comme le précise la norme, que les actions de la maîtrise de la qualité et de l'assurance qualité sont liées entre elles.

\section{Termes spécifiques à la modélisation numérique en génie civil}

Nous avons volontairement limité notre étude aux termes spécifiques à la modélisation des ouvrages et à l'interprétation qui en est faite par les utilisateurs de codes et les ingénieurs d'étude. Pour les termes courants, nous donnons, lorsqu'ils existent, les définitions proposées par les normes relatives à la qualité et par des dictionnaires, puis les références que nous avons relevées dans la littérature et enfin, soit nos propositions, soit des éléments permettant de contribuer à la discussion sur l'utilisation de ces termes.

\subsection{Vérification}

La norme stipule que la vérification est la « confirmation par examen et apport de preuves tangibles que les exigences spécifiées ont été satisfaites ».

Les interprétations du terme "vérification " different en fonction du type d'exigence auquel les différents auteurs se réfêrent. Nous avons relevé les références suivantes :

- «La vérification, consiste, stricto sensu, à s'assurer que les algorithmes et les formulations utilisées dans les codes ou la mise en cuvre du modèle, sont mathématiquement et méthodologiquement corrects [...]. La comparaison de solutions analytiques établies avec des résultats numériques, entre dans ce cadre » [PIG 95]. La vérification est donc considérée ici comme le premier niveau de contrôle d'un code ; il s'agit de contrôler l'absence de «bug " dans le code, la bonne programmation des équations ;

- «La procédure de vérification doit être scindée [...] selon trois notions différentes la validation du logiciel, la justification de la méthode complète de modélisation et l'assurance qualité de l'application particulière " [CIG 94]. Pour ces auteurs, la vérification a un sens beaucoup plus général. Il s'agit de vérifier l'ensemble du produit, donc l'ensemble des étapes, de la conception du code jusqu'à l'application particulière ;

- «La vérification consiste en un contrôle du modèle effectué par une comparaison entre les comportements observés et calculés » [MES 97]. 
Ces trois références nous montrent que l'interprétation de ce terme est variée. Compte tenu de la diversité des processus pouvant faire l'objet d'une vérification, nous proposons :

- que l'emploi du terme vérification soit assorti de précisions sur l'objet que l'on vérifie : on vérifie un processus ou des données, et il est nécessaire de spécifier ce que l'on vérifie (liste des objets);

- que les exigences auxquelles on se réfêre pour la vérification soit clairement énoncées.

La décomposition des activités de conception d'un code ou de réalisation d'une modélisation en processus peut permettre de se référer à une liste d'opérations, d'étapes par lesquelles il faut passer pour bâtir un code ou pour réaliser un calcul. Si l'analyse des processus est faite, il est possible de vérifier chacun des processus et la liaison entre les différents processus.

Toutefois, disposant d'autres termes pour évoquer les contrôles (voir notamment validation) et afin d'éviter toute confusion entre ces termes, nous proposons d'associer la vérification aux tests élémentaires du code et aux données du calcul.

La norme indique que la vérification est une " confirmation ». Il importe de savoir qui doit vérifier : la personne qui a écrit le code (ou réalisé la modélisation) ou une personne extérieure. On recommandera, en général, l'intervention d'une personne extérieure.

\subsection{Validation}

D'après la norme ISO 8402-2.1 [AFN 96], la définition de la validation est très proche de celle de la vérification, mais avec des nuances sur les «exigences ». Il s'agit "de la confirmation par examen et apport de preuves tangibles que les exigences particulières pour un usage spécifique prévu sont satisfaites » [AFN96]. Le petit Robert [ROB 97] définit le terme valide comme "qui présente les conditions requises pour produire son effet, qui n'est entaché d'aucune nullité ».

Nous avons recensé différentes références relatives à ce terme. Selon les auteurs, il s'agit de :

- « donner l'assurance que le logiciel donne la réponse correcte au problème qu'on a l'intention de résoudre à savoir une représentation idéalisée de la réalité " [CIG94] ;

- «montrer que le logiciel donne de bons résultats sur des cas jugés comme réels, c'est-à-dire se rapprochant plus de cas concrets [...]. Ce n'est plus seulement le logiciel que l'on valide, mais c'est aussi la personne qui l'utilise » [HOF 88] ; 
- "s'assurer que le modèle est conforme aux lois scientifiques et logiques" [PIG 95] ;

- "vérifier la justesse des équations physiques, de la précision et de la convergence des algorithmes de résolution » [GRE 95] ;

- «s'assurer que le modèle est capable de prédire de manière quantitative des phénomènes réels » [CAM 98] ;

- « contrôler un modèle vis-à-vis de situations connues " [LAM 73] ;

- « comparer par rapport à un banc d'essai issu d'une banque de tests » [CHA 88] ;

- «s'assurer que, pour un contexte d'utilisation déterminé, le logiciel modélise correctement les phénomènes physiques attendus » [GRC 99].

Nous constatons, dans ces définitions, que l'entité à valider, ainsi que le référentiel qui sert à la validation, diffèrent d'un auteur à l'autre :

- l'entité peut être :

- le code et sa programmation,

- le modèle : le modèle conceptuel (par exemple : phénomène mécanique pur, ou phénomène hydromécanique), le modèle géométrique (dimensions, conditions aux limites...), le modèle mécanique (comportement rhéologique, conditions initiales...) et le modèle numérique (méthode de résolution, discrétisation...).

Dans un cas, on valide un outil (code), dans l'autre cas on valide un choix pour la modélisation.

- le référentiel de validation peut être :

- les lois scientifiques (phénomènes physiques traduits sous forme d'équations mathématiques) ;

- une représentation idéalisée de la réalité (ceci inclut les solutions mathématiques et éventuellement des solutions proposées par des benchmarks) ;

- les phénomènes physiques observés in situ ou au laboratoire.

On remarquera que l'identification de l'entité validée est souvent difficile, car si le référentiel est une observation physique de terrain, la validation concerne à la fois le code et le modèle au sens large.

Etant donné la diversité des définitions, nous proposons de retenir un noyau commun qui consiste à définir la validation comme :

- la conformité du code aux « lois », à la "représentation idéalisée de la réalité » lorsqu'il s'agit de la validation d'un code ; 
- la conformité du modèle aux phénomènes physiques observés in situ ou au laboratoire lorsqu'il s'agit de valider le modèle.

Ces propositions ne nous semblent pas en contradiction avec la norme : pour chaque nouveau type de modélisation, il convient d'apporter des preuves que le modèle et le code employés sont satisfaisants. Par ailleurs, elles distinguent les deux notions, vérification et validation.

De plus, il nous paraît important que le terme validation soit assorti de précisions sur les critères (exigences) ayant conduit à la validation. Ces critères peuvent être différents selon les personnes qui procèdent à la validation. Dans le domaine de la consolidation de remblai par exemple, on peut envisager les exigences suivantes (liste non exhaustive) :

- le code doit reproduire le comportement mécanique et hydraulique ;

- le code doit reproduire le comportement mécanique et hydraulique de façon couplée, en milieu saturé ;

- le code doit reproduire le comportement mécanique et hydraulique de façon couplée, en milieu saturé et non saturé ;

- ou encore plus spécifique : le modèle doit reproduire à $10 \%$ le déplacement observé sur le terrain en un certain point.

\subsection{Justification}

Il s'agit, d'après les dictionnaires, "d'apporter les preuves de ce que l'on avance, de rendre légitime ce que l'on propose " [ROB 97]. C'est un terme très utilisé dans le domaine juridique. Ce terme n'est pas mentionné dans les normes relatives à la qualité.

Nous mentionnons ici deux références relatives à ce terme en géotechnique :

- la justification "d'un modèle " consiste à "donner les éléments permettant de prouver le bien fondé des hypothèses utilisées dans le modèle et de s'assurer de leur cohérence " [CAM 98] ;

- la justification « de l'ensemble de la procédure de calcul, de la méthode de calcul est sa capacité à représenter la réalité physique " [CIG 94].

On peut retenir de ces deux définitions que la justification consiste à apporter des preuves de la réponse correcte d'une modélisation pour le comportement d'un ouvrage donné. La conformité des résultats d'un calcul avec un comportement réel observé sur un ouvrage semblable ou lors des premières phases de construction de l'ouvrage étudié, peuvent constituer ces preuves. Toutefois, dans certaines 
circonstances " accidentelles », cet accord peut être assuré sans que le modèle soit correct. Aussi, il convient que ces preuves reposent également sur l'avis d'un expert et dans une certaine mesure sur une pratique traditionnelle.

Les définitions divergent, en général, sur les entités que l'on cherche à justifier. On peut, par exemple, justifier :

- une procédure globale de calcul. Dans ce cas, compte tenu de la limite des modèles, une procédure justifiée ne s'appliquera qu'à une classe de problèmes qu'il convient de définir précisément ;

- les paramètres et le modèle de comportement d'un matériau par analyse des essais de laboratoire ou des essais in situ. On vérifie ainsi que le modèle est représentatif des phénomènes observés en champ de contrainte homogène et/ou sur chemin de contraintes particulier. Il s'agit alors d'une composante de la procédure globale.

Pour différencier le terme justification du terme validation, on peut suggérer que :

- la justification est le processus qui consiste à apporter des preuves ; ce terme a donc une connotation juridique. Il est d'ailleurs très utilisé dans ce domaine ;

- la validation est le résultat de ce processus : tout est en ordre et fonctionne.

\subsection{Identification}

Ce terme est utilisé par les qualiticiens sous une forme générale pour représenter un produit, un processus, un facteur de qualité, un besoin... Il va de pair avec la traçabilité : aptitude à garder en mémoire et à retrouver l'historique.

Cette conception est assez éloignée des préoccupations du génie civil. En effet, nous n'avons pas trouvé de référence définissant «l'identification » en génie civil. Il semble pourtant qu'il existe un consensus parmi les géotechniciens pour considérer l'identification comme la détermination de certains paramètres (paramètres rhéologiques) nécessaires à la modélisation à partir de résultats expérimentaux en laboratoire exclusivement pour certains auteurs, d'essais de laboratoire et in situ pour d'autres auteurs. Cette détermination peut être simple et explicite (par exemple l'identification du module d'Young d'un matériau se fera en linéarisant la courbe effort-déformation), mais elle peut être plus complexe et nécessiter des ajustements par calage (l'identification se fera par calage). 


\subsection{Calage}

Il n'existe pas de définition normalisée pour le terme calage et les définitions proposées par les dictionnaires sont assez éloignées des préoccupations de modélisation. On peut cependant retenir de ces définitions qu'il s'agit « d'un réglage optimal ». Dans le domaine de la géotechnique, ce terme est couramment utilisé :

- selon Cambou, «il s'agit d'un processus particulier qui peut être utilisé dans un processus d'identification lorsque la complexité du modèle et le niveau d'information expérimentale empêchent d'avoir une évaluation explicite et unique de l'ensemble des paramètres du modèle. Le calage consiste à choisir les valeurs numériques des paramètres de telle sorte que les résultats des simulations soient en conformité avec les aspects jugés essentiels du comportement mesuré » [CAM 98] ;

- selon Piguet, «le calage [...] consiste à ajuster au mieux les valeurs des paramètres introduits comme données dans les modèles (et pour lesquelles règnent une imprécision ou une incertitude, voire une ignorance complète) en faisant en sorte que les résultats des modèles issus des calculs soient en conformité avec les aspects, jugés essentiels, du comportement réel observé (et mesuré). Le calage peut aussi être étendu à la pertinence du choix des variables ou des critères retenus pour l'interprétation des résultats. Il s'apparente donc à un problème inverse » [PIG 95].

Ainsi, le calage apparaît comme une phase de tâtonnements où l'on fait varier les paramètres d'un modèle. Le calage considéré comme optimal, conduit à "l'identification des paramètres ». Les points de vue peuvent diverger ensuite selon la nature des mesures sur lesquelles portent les comparaisons (mesures in situ et/ou essais de laboratoire simples et/ou complexes) et selon les entrées du modèle sur lesquelles l'utilisateur va agir en fonction de ces comparaisons. Classiquement, pour un géotechnicien, le calage concerne les paramètres rhéologiques du sol. Mais il peut impliquer également tous les choix de la modélisation : état de contrainte initial, conditions limites, conditions d'interface, discrétisation de l'espace, type d'éléments finis, modèle rhéologique, jeu de paramètres du modèle rhéologique, paramètre de déconfinement pour la convergence d'un tunnel...

\subsection{Erreur, Incertitude, Approximation}

L'erreur, selon un qualiticien, résulte d'une défaillance technique, organisationnelle ou humaine. Dans une modélisation numérique, on peut concevoir une erreur au sens de la norme (défaillance $=$ " fonction non remplie alors qu'elle était prévue »). Mais ce terme est généralement employé pour désigner :

- l'approximation dans la méthode de résolution, la référence étant une solution analytique découlant directement des lois scientifiques ; 
- ou l'incertitude dans la modélisation, la référence étant le résultat d'essais [REY 99].

Nous constatons ainsi qu'il n'est pas approprié d'appeler " erreur » la distance entre le modèle et les résultats d'essais, car cette différence peut avoir des origines diverses prêtant à confusion. Cependant, le terme « erreur » semble unanimement adopté par les spécialistes de cette différence entre modèles et essais. Il conviendrait, peut-être, pour préciser ces termes, de se référer aux normes relatives à la mesure.

On distinguera :

- une erreur a priori qui ne nécessite pas un calcul numérique préalable et qui peut fournir un majorant de l'erreur pour une discrétisation donnée ;

- une erreur a posteriori qui se réfêre à un résultat de calcul numérique préalable.

En toute rigueur, la performance d'une modélisation sera évaluée quantitativement par un estimateur mesurant la distance entre la solution exacte des lois scientifiques (ou des résultats d'essais) et le résultat du calcul, et plus qualitativement par des indicateurs analysant le résultat numérique (ex. : amplitude de la discontinuité des contraintes) sans référence à une solution exacte ou un résultat d'essai. Il s'avère que la distinction entre ces deux notions n'est pas clairement établie et que les deux termes (estimateurs et indicateurs) ne sont pas toujours utilisés à bon escient.

\subsection{Benchmark, cas test, banc d'essai, prévisions, prédictions}

Ces différents termes relèvent tous de la comparaison, une comparaison qui peut concerner un code et/ou un modèle, avec :

- une solution analytique ;

- une expérimentation de laboratoire, un essai in situ, un ouvrage ;

- d'autres codes.

Par ailleurs, les modalités des confrontations varient. Les comparaisons sont effectuées en «aveugle" ou non. Pour le calcul d'ouvrages, Lambe [LAM 73] distingue les calculs effectués :

- avant l'ouvrage (prédiction de catégorie A) ;

- pendant la construction de l'ouvrage ; on dispose de certaines mesures sur le site pour une prédiction de catégorie $\mathrm{Bl}$. On ne dispose pas de ces mesures pour une prédiction de catégorie $B$;

- après la construction de l'ouvrage ; on dispose de certaines mesures sur le site pour 
une prédiction de catégorie $\mathrm{Cl}$. On ne dispose pas de ces mesures pour une prédiction de catégorie $C$. Enfin, les données fournies aux participants de telles confrontations sont très diverses. Le maillage, les conditions aux limites, les conditions initiales, les paramètres rhéologiques, les chargements sont imposés explicitement ou il convient d'interpréter des informations afin de déterminer ces différents paramètres.

Pour évoquer tous ces types de tests, nous disposons de plusieurs termes, qui ne sont pas synonymes: benchmarks, cas tests, référentiels, banc d'essais, prévisions, prédictions. Le fait d'associer un terme à chaque type de tests permettrait de préciser les conditions et ainsi leurs objectifs. En effet, ceux-ci different non seulement en fonction des modalités de la confrontation, mais aussi dans les objectifs : le test peut consister à comparer des codes entre eux ou à témoigner de la capacité des équipes à modéliser un ouvrage. Actuellement, en l'absence d'éléments suffisants sur l'emploi de ces termes notamment dans des secteurs autres que celui du génie civil, nous différons toute proposition de définitions plus précises.

\subsection{Qualification}

Pour certains auteurs, la validation fait état de comparaisons avec des modèles physiques, alors que la qualification utilise comme référence une application en conditions réelles. Toutefois, on retiendra un sens plus général, celui de la norme ISO 8402-2.1 [AFN 96], qui considère le processus de qualification comme un "processus démontrant qu'une entité est capable de répondre aux exigences spécifiées ». La qualification reconnaît donc, après vérification (confirmation par examen que les exigences spécifiées ont été satisfaites) ou validation (confirmation par examen que les exigences particulières pour un usage spécifique prévu sont satisfaites), qu'une entité possède tous les gages de l'assurance qualité. La qualification est alors considérée comme l'étape ultime de contrôle : « la qualification constitue une vérification globale du produit fini » [VOU 88].

Mais, dans ce cas, l'ambiguité évoquée avec les termes "vérification » et "validation" subsiste : que contrôle-t-on ? le code ? le modèle ? l'utilisateur ? Aussi, il nous semble essentiel de distinguer ce qu'on qualifie. Sur la base d'une formation et/ou d'un test, on qualifiera une personne. Sur la base d'une expérience, d'un MAQ, on qualifiera un code, un bureau d'étude ou la note de calcul qu'il produit.

\section{Conclusion}

La qualité d'une étude exige, en tout premier lieu, de la part des différents partenaires, une parfaite maîtrise des termes liés à toutes les étapes d'une opération 
de modélisation numérique d'un ouvrage. Dans un système qualité, les concepteurs de codes, les praticiens de ces codes, les chercheurs, les destinataires des notes de calcul se doivent de pratiquer un langage commun et ont besoin de points de repère.

La confusion qui règne actuellement vient du fait que les acteurs de la modélisation ont généralement procédé à un découpage de leurs activités en associant à chacune des opérations un terme courant de la modélisation numérique. Toutefois, les préoccupations de ces intervenants étant de natures diverses, ceci a conduit pour certains termes à des contenus sémantiques variés.

Pour un certain nombre de termes, nous avons proposé une définition correspondant aux éléments communs aux différents auteurs. Pour d'autres, nous proposons que les termes soient accompagnés de précisions sur leur domaine d'application.

Cette mini synthèse sur la terminologie a pour objectif de contribuer à une plus large réflexion sur le bon usage des divers termes utilisés lors d'une modélisation. Elle a été réalisée à partir d'éléments bibliographiques mais, comme les suggestions dans ce domaine sont peu nombreuses et parfois contradictoires, elle devra être poursuivie et complétée avant de se concrétiser sous la forme d'un lexique qui aurait l'assentiment de tous.

\section{Bibliographie}

[AFN 96] "Gérer et assurer la qualité - Qualité et efficacité des organisations ». Recueil de Normes Francaises 1996, AFNOR.

[AFZ 88] M. AfZALI, M. Hervé, "Qualité et validation des progiciels de calcul », Revue Française de Mécanique, $n^{\circ} 1988-3,1988$.

[CAM 98] B. Cambou, Communication privée, 1998.

[CHA 88] C. ChARbonnier, "Assurance-qualité, des développements ou validation a posteriori des progiciels de calculs de structures ", Revue Française de Mécanique, n 1988-3. [CIG 94] «Computer software for dams : Validation, comments and proposals - Logiciels de calcul des barrages : Validation, réflexions et propositions », Bulletin 94, ICOLD - CIGB, 1994.

[DEF 96] V. DEFOURNY, D. NoYÉ, « Du bon usage des mots de la qualité, les principaux termes : définitions et commentaires », INSEP édition, Ministère de l'industrie, de la poste et des télécommunications.

[GRC 99] Groupe C, sous-groupe GT8, « Exigences assurance qualité pour la mise en œuvre de codes de calcul », Spécification 910 SP06 24.

[GRE 95] Les Géomatériaux: Avancées récentes en calcul d'ouvrages, tome 3, Hermès, 1995. 
[HOF 88] A. Hoffmann, A. Combescure, A. Millard, "Validation des progiciels de calcul en milieu industriel : une expérience, celle de CASTEM ", Revue Française de Mécanique, $\mathrm{n}^{\circ}$ 1988-3.

[LAM 73] T.W. LAMBE, «Prediction in soil engineering », Géotechnique 23, n², pp. 149202, 1973.

[MES 97] MESTAT P., «Practical applications of modelling and numerical methods in geotechnical engineering", oct. 1997, Rapport interne du Comité Technique Régional Européen $n^{\circ} 7$ (ERTC7), 1997.

[PIG 95] PIGUET J.P., «Ingénierie en mécanique des roches : de nouveaux progrès en informatique comme moyen de traiter l'empirisme », Keynote lecture - Proc. of the 8th. Congress on Rock Mechanics (ISRM), Balkema, Tokyo, Japon, 1995.

[REY 99] M. REYNIER, «Indicateurs de qualité de modélisations éléments finis : Calage à partir des données mesurées ", mini symposium AUGC-AFPC, $2^{\mathrm{e}}$ congrès de l'AUGC, Poitiers, 6-7 mai 1999.

[ROB 97] Le petit Robert, Dictionnaire de la langue Française.

[SOU 88] J.C. SourisSEAU, J. LoCATELl, «Validation des codes internes et externes à l'aérospatiale ", Revue Française de Mécanique, n 1988-3.

[VOU 88] F. Voullloux, "L'assurance qualité critère de choix d'un logiciel», Revue Française de Mécanique, $\mathrm{n}^{\circ}$ 1988-3. 